\title{
Startle epilepsy
}

INSERM

\section{Source}

INSERM. (1999). Orphanet: an online rare disease and orphan drug data base. Startle epilepsy. ORPHA:166427

Startle epilepsy is a rare neurologic disease characterized by frequent and spontaneous epileptic seizures (frequently with symmetrical or asymmetrical tonic features) triggered by a normal startle in response to a sudden and unexpected somatosensory (most frequently auditory) stimulus. Falls are common and can be traumatic. In most cases, the disease is associated with spastic hemi-, di-, or tetraplegia and intellectual disability. 